\title{
La débitmétrie en milieu industriel
}

La plupart des procédés industriels requièrent des appareils de mesure pour déterminer les débits de divers fluides. Ces appareils sont destinés soit au comptage des quantités de fluide les traversant, soit au pilotage et au contrôle d'installations.

L'utilisation de débitmètres en milieu industriel pose de nombreux problèmes dont les principaux sont les performances, l'étalonnage et le coût des appareils mis en auvre. La deuxième journée de ce Colloque est consacrée à ces trois aspects.

Certaines applications nécessitent le développement et la qualification d'instruments originaux dont les fonctionnalités techniques n'existent pas dans le domaine commercial ou même dans les laboratoires. Cinq communications relèvent de cet aspect développement et qualification de techniques nouvelles. Elles abordent (I) la mesure et le contrôle de très faibles débits pouvant descendre jusqu'au $\mathrm{cm}^{3} /$ an avec de nombreuses applications en chimie, agriculture, cosmétique, médecine, pharmacie et autres techniques de pointe, (2) la débitmétrie en hydrogène liquide utilisée dans les applications aérospatiales, (3) la débitmétrie en écoulement diphasique de propane, (4) la débitmétrie dans les écoulements multiphasiques par la résonance magnétique nucléaire. (5) la débitmétrie dans les réseaux de distribution de vapeur saturée pour le chauffage urbain. On remarquera que trois des cinq communications précédentes sont relatives aux écoulements diphasiques montrant bien l' importance des problèmes de débitmétrie dans ces écoulements.

Les problèmes de coût sont essentiels pour qu' un instrument soit compétitif sur le marché international. Les constructeurs d'équipements de débitmétrie sont donc à la recherche de systèmes simples, précis et fiables. Deux exposés sont consacrés à de telles techniques: (1) l'oscillateur fluidique qui, bien que simple, met en jeu des écoulements complexes mais très structurés, (2) le débitmètre à vortex qui est le siège de tourbillons organisés dont la visualisation permet une amélioration de la compréhension.

Une dernière communication traite des moyens d'étalonnage des débitmètres à gaz, sujet important tant par ses retombées industrielles que par ses implications au laboratoire.
L'ensemble de ces exposés ont fait ressortir les points suivants :

1. Le besoin d'une certaine créativité pour développer ou appliquer des principes nouveaux pour permettre des mesures impossibles jusqu'à ce jour ou pour simplifier ou remplacer des techniques existantes.

2. La nécessité de considérer l'ensemble de la chaîne de mesure allant de l'interaction du capteur avec l'écoulement au traitement du signal en passant par l'électronique analogique et numérique.

3. Le passage obligé par une étape d'industrialisation et de commercialisation des dispositifs mis au point dans les laboratoires cela ne pouvant relever que d'une volonté politique clairement exprimée par les ministères concernés et accompagnée par des aides significatives et fiables.

4. L'intervention technique constante au niveau des groupes de travail français et internationaux élaborant les normes.

5. Le maintien d'équipes de haute qualité pour l'étalonnage des débitmètres pour les liquides et les gaz.

6. Le développement de bancs d'étalonnage français pour les écoulements diphasiques analogues à ceux existant au $N E L$.

Un des grands mérites de cette journée a été de rassembler des ingénieurs et des chercheurs de secteurs industriels très variés. Souhaitons que ces contacts se poursuivent et que la SHF en reste le moteur efficace et constant.

J.M. DELHAYE

Commissariat à l'Energie Atomique Professeur à l'Ecole Centrale Paris

Dernière minute.

Monsieur J.P. HUFFENUS devait présider la seconde demi-journée du colloque. Comme il était gravement atteint par la maladie, son ami M. J.M. DELHAYE l'a remplacé. Nous avons eu la douleur d'apprendre que la maladie l'a emporté, avañt même que ces lignes ne soient imprimées. La Société Hydrotechnique de France lui rendra hommage dans les colonnes de la SHF par ailleurs. 


\section{Discussions de la $2^{\mathrm{e}}$ journée}

\section{Mesure de très faibles débits en écoulements monophasiques par Y. LECOFFRE}

- Question (M. WESFREID) :

S'il s'agit d'un milieu poreux et si le nombre de Reynolds est de l'ordre de l'unité, la loi de DARCY s'exprime par une relation quadratique du type $\Delta \mathrm{p}=\mathrm{aV}+\mathrm{bV}^{2} \ldots$ Ce terme quadratique a-t-il été pris en compte?

\section{- Réponse (M. LECOFFRE) :}

Tout d'abord le nombre de Reynolds est supérieur à 1 , de l'ordre de 5 à 6 . Ensuite nous ne sommes pas en présence d'un milieu poreux puisque le système se réduit à un seul capillaire de $2 \mathrm{~m}$ de longueur et d'un diamètre équivalent de $100 \mu \mathrm{m}$. Un milieu poreux de par le nombre de pores permettrait des débits trop importants.

- Question (M. WILlemeTZ) :

Quels sont les risques d'obturation de ces canaux de très faibles diamètres?

\section{- Réponse (M. LECOFFRE) :}

Les fluides utilisés sont en général assez propres. Cependant on intercale en amont un filtre, dont le diamètre capillaire est de l'ordre de $5 \mu \mathrm{m}$.

La protection contre les dépôts se fait par des contrôles réguliers de l'état chimique et de la température (ce qui est nécessaire d'autre part puisque la loi de débit dépend de la viscosité).

\section{- Question (M. Delhaye) :}

Comment assure-t-on l'étanchéité entre les spires du ressort et les parois et entre les spires elles-mêmes ?

- Réponse (M. LECOFFre) :

Un appareil diffuseur de parfum, par exemple, doit rester très peu cher (de l'ordre de $10 \mathrm{~F}$ ); aucune technologie avancée ne pourra donc être utilisée.

La paroi externe est en élastomère et épouse la forme des spires. Elle permet une meilleure adhérence entre les spires et la paroi, et entre les spires elles-mêmes.

\section{- Question (M. CAZENAVE) :}

Le dispositif décrit peut être utilisé jusqu'à un débit de $1 \mathrm{~cm}^{3} / \mathrm{an}$. Pensez-vous qu'il existe un « marché » pour des débits inférieurs à cette limite ?

\section{- Réponse (M. LeCOFFRE) :}

Cette limite dépend du fluide utilisé ; dans le cas du gaz le débit minimum est en effet $1 \mathrm{~cm}^{3} /$ mois.

On peut envisager de descendre en dessous d'un diamètre équivalent de $30 \mu \mathrm{m}$ dans l'usinage de la pièce et ainsi d'atteindre les $91 \mathrm{~cm}^{3} /$ an pour l'eau.

Les marchés liés à ces faibles débits sont la parfumerie, la médecine, la biologie et l'agriculture.

- Question (M. Kerevan) :

Comment sont étalonnés les appareils ?

\section{- Réponse (M. LECOFFRE) :}

L'étalonnage est toujours délicat mais réalisable.

Pour les débits de l'ordre de $1 \mathrm{~cm}^{3} / \mathrm{min}$ on utilise une méthode de pesée. Pour les débits de l'ordre de $1 \mathrm{~mm}^{3} / \mathrm{min}$ le problème est plus délicat : on étalonne avec un tube capillaire dans lequel on injecte une bulle dont on mesure la vitesse de déplacement.

En général le contrôle de la température doit être effectué avec précautions.

\section{Mesure et contrôle des débits pour la perfu- sion parentérale par P. REBours}

\section{- Question (M. Cazenave) :}

La précision devant être d'autant plus grande que le débit est faible, n'y a-t-il pas une solution consistant à utiliser des liquides plus dilués ?

\section{- Réponse (D. Cazala) :}

Aux Etats-Unis le nombre de pharmaciens par hôpital est beaucoup plus élevé et les solutions diluées arrivent prêtes à être utilisées par les infirmières. On est de toute manière gêné par la quantité de liquide nécessaire (on injecte déjà de 11 à 1,5 1 par jour rien qu'avec des produits concentrés).

Commentaires :

- Méthode de perfusion par gravité : il peut y avoir des variations de 8 à 40 gouttes/min et la précision est très faible sur le débit.

- Utilisation de seringue : convenable sauf pour les très faibles quantités, pour les prématurés $(0,1$ à $2 \mathrm{ml} / \mathrm{h})$ ou pour les injections de produits toxiques.

Problème de l'alarme de dysfonctionnement :

* Combien de temps faudra-t-il pour détecter une obturation du cathéter?

* Le fluide exerce une pression sur l'occlusion et si on débouche brusquement le canal, les conséquences peuvent être dramatiques pour le malade.

Seuls des moyens de détection basés sur la mesure de pression sont disponibles, il faudrait des moyens de détection basés sur la mesure du débit (qui soient peu coûteux, une pompe à perfusion coûtant de 8 à $10 \mathrm{kF}$ ).

- Question (M. DelhaYE) :

Existe-t-il des normes sur la précision et la régularité des débits injectés par voie parentérale?

- Réponse :

La précision doit être de $\pm 1 \%$ sur le débit en linéaire, ces normes sont juste établies pour la sécurité.

- Question (M. LECOFFRE) :

Quel est à votre avis l'avenir des diffuseurs intracorporels ? 


\section{- Réponse :}

Des techniques sont en développement pour l'insuline, par exemple, qui nécessitent une injection interne mais aussi variable dans le temps.

Des recherches sont aussi en cours sur l'injection d'antalgiques par voie rachidique, veineuse où à l'aide d'un patch sous-cutané.

- Question (M. LECOFFRE) :

Quelles sont, à votre avis les limitations principales à des systèmes implantables à l'intérieur du corps humain ?

- Réponse :

Il faut étudier le problème de diffusion du produit suivant l'endroit de l'injection (une diffusion sous-cutanée peut par exemple être mauvaise).

Le système de remplissage du dispositif doit rester facile.

Le problème du rejet du dispositif par l'organisme du patient reste incontournable.

\section{Débitmétrie en hydrogène liquide par} A. MEMPonteIL (présentation : E. HerviEU)

\section{- Question (M. BravaIS) :}

Pour quelle raison utilise-t-on de l'argon dans le débitmètre Coriolis ?

\section{- Réponse :}

L'argon est un gaz rare qui a été choisi par le constructeur pour des raisons de non-corrosion. Des impératifs technologiques et économiques ont sans doute conduit les constructeurs à préférer ce gaz à l'hélium qui est lui plus stable.

- Question (M. BravaIS) :

Est-ce qu'il est envisagé d'étendre la gamme de débit $(200 \mathrm{~g} / \mathrm{s})$ ?

- Réponse :

La valeur limite du débit est imposée par la géométrie de la section d'essais. Des débits plus élevés ne sont pas prévus dans le programme actuel.

\section{- Question (M. LECOFFrE) :}

$\mathrm{Ne}$ pensez-vous pas qu'il soit possible d'utiliser une mesure déprimogène présentant un col réglable?

\section{- Réponse :}

L'introduction de pièces en mouvement au contact de l'hydrogène est toujours très délicate et doit être évitée au maximum.

De plus dans le cas de l'étude de transitoires rapides les problèmes de dynamique rendent le débitmètre inutilisable.

\section{Oscillateur fluidique : application à la débit- métrie des écoulements monophasiques par J.E. WESFREID}

\section{- Question (M. STRZELECKI) :}

Est-il possible d'augmenter la dynamique d'un tel débitmètre fluidique ?

\section{- Réponse (M. WESFREID) :}

Les débitmètres étudiés en laboratoire ont en général une mauvaise dynamique. En revanche le débitmètre industriel équivalent peut mesurer des débits jusqu'à $6 \mathrm{~m}^{3} / \mathrm{h}$. Je suis convaincu que SCHLUMBERGER (industriel qui développe le débitmètre) peut atteindre des débits maximums de $12 \mathrm{~m}^{3} / \mathrm{h}$.

- Question (M. STRZEVECKI) :

Avez-vous étudié l'influence de perturbations périodiques ou transitoires sur ce débitmètre?

- Réponse (M. WeSFreid) :

Dans le débitmètre à vortex il existe deux mécanismes d'instabilités qui sont couplés entre eux. Lorsque l'on utilise un jet confiné, on est beaucoup moins sensible à l'un d'entre eux.

Le prototype industriel est lui moins sensible aux perturbations en amont de l'écoulement.

- Question (M. Vernier) :

Avez-vous étudié la caractéristique $\Delta \mathrm{P}=\mathrm{f}(\mathrm{Q})$ des appareils?

Malgré l'écoulement laminaire, n'est-elle pas non linéaire ? Le problème se pose pour le couplage pressions-débits avec l'installation où l'appareil est monté.

\section{- Réponse (M. WESFREID) :}

Ces caractéristiques ont été obtenues par SCHLUMBERGER, je n'en ai pas connaissance.

\section{- Question (E. HerviEu) :}

L'utilisation de méthodes d'analyse du signal plus sophistiquées (temps-fréquence, ondelettes) que l'analyse de FOURIER classique ne pouvait-elle pas apporter des informations plus riches concernant le transitoire d'établissement des oscillations?

\section{- Réponse (M. WESFREID) :}

Le problème de l'établissement des oscillations est tout à fait équivalent au problème de l' " attaque de la note " en musique, il y a participation de termes non linéaires. On peut effectivement faire une étude plus poussée, tempsfréquence par exemple mais les informations les plus importantes pour nous résident dans le terme de fréquence nulle et le terme du premier harmonique.

\section{Débitmétrie des gaz : Moyens d'essai et éta- lonnages par P. KEREVAN (GDF)}

- Question (M. DelhaYe) :

Quelles sont les relations de GDF avec le K-Lab de Korstoe (Norvège) ?

- Réponse (M. Kerevan) :

Les chercheurs du K-Lab utilisent la méthode gravimétrique pour étalonner leur débitmètre ; les confrontations de cette méthode avec la méthode PVT, utilisée par GDF, pour une tuyère sonique a donné de très bons résultats.

\section{Analyse de l'écoulement au voisinage d'un débitmètre à vortex pour améliorer la préci- sion de comptage en milieu industriel, par Alain STrzelecki, ONERA CERT Toulouse}

- M. Delhaye souligne la qualité des visualisations de l'écoulement que M. StRZELECKI a présentées. Il fait 
remarquer que de telles visualisations sont très importantes en mécanique des fluides et qu'elles devraient être l'étape préliminaire à toute étude.

\section{- Question de M. WeSFreId (ESPCI-Paris) :}

«Les perturbations créées par un coude sont modélisées par une rotation. Ne faudrait-il pas plutôt faire intervenir plusieurs rotations ? »

M. STRZELECKI précise que la perturbation créée par un coude se décompose en deux parties:

- les tourbillons de Dean qui apparaissent à la sortie du coude ;

- une dissymétrie du profil de vitesse.

En revanche, si on place en série sur la conduite deux coudes non coplanaires, alors on génère bien une rotation dans l'écoulement.

Mais on trouve aussi des rotations à l'aval de certains organes sur les conduites industrielles (détendeur par exemple), ce qui explique l'intérêt d'étudier l'influence des rotations sur les débitmètres à vortex.

\section{Débitmétrie d'un écoulement diphasique de propane par Eric HERVIEU, CEA-CENG-STI Grenoble}

\section{- Question de M. Bravais (L'Air Liquide) :}

«Dans la mesure où l'entraînement de bulles et la formation de vortex dépendent fortement de la géométrie, quelle est la validité de cet essai qui met en œuvre un réservoir de très faible volume?»

M. HERVIEU explique que l'objectif n'était pas de simuler le cas industriel mais de dégager les mécanismes physiques qui interviennent dans la vidange du réservoir.

De plus, le CENG contenant des installations nucléaires, il se posait un problème de sécurité. Afin d'éviter d'avoir à déclarer l'installation comme ICPE (Installation Classée pour l'Environnement), l'étude a été limitée à un réservoir de 91 pressurisé à 18 bar.

Enfin M. HeRVIEU ajoute qu'aucune étude à l'échelle réelle n'est prévue.

M. DelhaYe précise que cette installation est unique au monde car elle est instrumentée et qu'il est possible de faire des visualisations de l'écoulement.

\section{- Question de M. Cazenave :}

«L'étude présentée s'inscrit dans une chaîne de modélisation des risques de stockage et transport d'hydrocarbures sous pression. Comment va s'organiser la suite de cette chaîne ?»

M. HERVIEU répond que cette étude s'inscrit effectivement dans le cadre d'une étude plus globale dont les différents éléments sont traités par différents laboratoires.

Le CENG n'est pas chargé de l'aspect sûreté des réservoirs de stockage. Il fournit les résultats sur la vidange des réservoirs à d'autres personnes qui calculent alors l'expansion du nuage et l'explosion.

M. HeRviEu précise que le CENG a actuellement en cours un autre contrat sur l'étude de la granulométrie du jet.

\section{- Question de M. LespinaRd :}

"Que sait-on de l'indépendance du moulinet par rapport à la distribution du taux de vide ? »
L'influence de la répartition des phases sur le moulinet n'a pas été testée, il a été supposé qu'il réagissait comme avec un écoulement homogène.

Par contre, le densitomètre a été optimisé pour être indépendant de la répartition des phases.

\section{Débitmétrie multiphasique par résonance magnétique nucléaire par Sylvain JAVELOT, ESPCI Paris}

\section{- Question de M. Fautrelle (ENSHMG) :}

«Quel est le degré de localité de la mesure? »

M. JAVELOT répond que les mesures résultent de moyennes faites sur la zone de mesure qui est approximativement un cube de $5 \mathrm{~cm}$ d'arête.

\section{- Question de M. LeSPINARD (INPG) :}

«Comment localisez-vous la zone de mesure, car l'aimant est beaucoup plus gros? "

L'aimant est relativement grand mais il a une zone d'homogénéité faible où sont placées les bobines. La zone de contrôle est un cylindre dont la hauteur est celle des bobines et la section droite est celle de la conduite.

- Question de M. Delhaye (CENG) :

«Quelle est la nature de la paroi ? Peut-elle être métallique ?»

La paroi utilisée était en verre.

Elle peut être en céramique, plexiglas, ... mais pas en métal.

- Question de M. DelhaYe :

«Dans les mesures de débits, faut-il supposer un certain profil de vitesse? »

La réponse est négative.

M. Delhaye fait remarquer que cette méthode permet donc de mesurer des débits à l'aval de singularités.

\section{- Question de M. Delhaye :}

«Les vitesses obtenues en écoulement monophasique liquide doivent correspondre à des artefacts. Est-ce exact?»

M. JAVELOT confirme et pense que ces artefacts sont dus au temps de montée du signal.

\section{- Suggestion de M. DelhaYe :}

« Il serait intéressant de faire des essais en écoulement annulaire dispersé et pas seulement dans des écoulements à bulles ou à poches. La densité de probabilité de vitesse présenterait alors deux bosses qui correspondraient au débit de film et au débit des gouttes. "

M. JAVElot précise que la technologie actuelle ne permet pas de traiter ce type d'écoulements.

- Question de M. ? :

«Y a-t-il un problème de sensibilité à certains types d'atomes de cette méthode?»

Les problèmes de sensibilité sont liés au nombre de particules qui réagissent.

Par exemple, dans le cas des essais eau-fréon liquide, la molécule de fréon étant beaucoup plus grosse que la molécule d'eau, il y a donc dans la zone de mesure moins de molécules de fréon qui répondent que de molécules d'eau. 
Pour le fréon, il faut donc faire des mesures sur un temps plus long ou bien pour les mesures instantanées il faut sacrifier le rapport Signal/Bruit.

Lorsque la proportion de fréon devient inférieure à $10 \%$, il n'est plus possible de faire des mesures.

\section{- Question de M. LeSPINARD :}

«Peut-on utiliser cette méthode comme densitomètre?»

La réponse de M. JAVELOT est positive.

M. Delhaye note que l'intérêt de cette méthode réside dans le fait que l'on mesure directement le nombre d'atomes, on ne passe pas par une grandeur intermédiaire.

- Question de M. LeSPINARD :

"Quelles sont les autres molécules qui répondent?"

"Le fluor est-il privilégié ? "

Toutes les molécules qui ont un spin non nul, soit 80 à $90 \%$ des éléments de la table, sont utilisables.

L'intérêt du fluor est que sa fréquence de résonance est relativement proche de celle du proton, ce qui minimise les problèmes instrumentaux.

\section{- Précision de M. Leblond (ESPCI) :}

Certains noyaux sont peu visibles, ce qui allonge la durée des mesures.

Les plus intéressants sont : le fluor, le proton, le deutérium, le chlore et le potassium.

On peut noter qu'il existe différents protons : le proton de l'eau (liaison $\mathrm{OH}$ ) et le proton des hydrocarbures (liaisons $\mathrm{CH}_{2}$ et $\mathrm{CH}_{3}$ ) ne résonnent pas à la même fréquence.

Il est possible de les séparer en utilisant un champ magnétique assez élevé.

Pour l'expérience qui a été présentée, le champ utilisé était relativement bas $(0,12 \mathrm{~T})$ pour des problèmes de coût. $\mathrm{Si}$ on augmente ce champ d'un facteur 10 , il devient possible de séparer les protons de l'eau de ceux de l'huile par exemple.

\section{- Question de M. Delhaye :}

« Pourrait-on mesurer les débits des phases et la fraction volumique en régime transitoire?"

M. JAVELOT déclare que c'est possible mais que l'on aura accès seulement aux valeurs moyennes sur la zone de mesure. On n'aura pas par exemple la distribution de vitesse à l'intérieur de la conduite.

\section{Mesures de débits dans les écoulements dipha- siques de vapeur saturée par Yves Lecoffre YLEC, Consultant - St Ismier}

\section{- Question de M. LeSPINARD (INPG) :}

«Que peut-on dire des pertes de charges dans l’Opti$\operatorname{mix} ? »$

M. LECOFFRE répond qu'elles sont optimisées.

En effet, afin d'obtenir la granulométrie souhaitée, il faut régler la dimension de l'échelle de macromélange (c'est-à-dire la taille des viroles du $1^{\text {er }}$ étage) et celle de l'échelle de micromélange. L'appareil est optimisé de manière à ce que les pertes de charge soient les plus faibles compatibles avec la granulométrie souhaitée.

- Question de M. Aтtiach :

"Quel est le temps de réponse de l'appareil ? "

C'est le temps de passage du liquide dans l'appareil.
- Question de M. ? :

«Quelles granulométries peut-on obtenir? »

La granulométrie possible est très vaste.

Le CENG a effectué des essais eau-huile avec une granulométrie de 1 à $5 \mathrm{~mm}$ de diamètre.

M. LECOFFre a effectué lui-même des essais avec de l'asphalte avec des grains de $8 \mu \mathrm{m}$.

Il pense qu'on peut descendre jusqu'à $1 \mu \mathrm{m}$.

- Question de M. Kerevan (Gaz de France) :

«Pour le Diflux, comment fonctionne le système de régulation de la tuyère à col réglable ? "

Il y a 4 tuyères en parallèle.

On impose une pression de $10^{4} \mathrm{~Pa}$ au col qui est régulé par un actuateur hydraulique dont le temps de réponse est 3 à $4 \mathrm{~s}$.

- Remarque de M. Lemonnier (CENG) :

Ce type de mélangeur est très intéressant en chimie car pour les réactions à cinétique rapide, il est important de pouvoir régler les échelles de mélange.

\section{TABLE RONDE : Les problèmes non résolus dans la mesure et le contrôle des débits en conduite}

Participants :

Y. FAutrelle: Professeur à l'INPG - Directeur de l'ENSHMG.

H. ANDRÉ: Rapporteur général de la $1^{\text {ère }}$ journée du colloque.

J.M. Delhaye: CEA-CENG-STI - Professeur à l'Ecole Centrale Paris.

G. LESPINARD : Professeur à l'INPG.

J.L. Guillermou : Société Parisienne des Eaux-Paris.

Sous la présidence de M. FAutrelle, la discussion s'organise en 3 parties :

1. Synthèse de la $1^{\text {ère }}$ journée du colloque par M. ANDRÉ.

2. Synthèse de la $2^{\mathrm{e}}$ journée par M. Delhaye.

3. Discussion sur les perspectives.

\section{Synthèse de la $1^{\text {re }}$ journée: Contrôle des débits dans les réseaux urbains d'adduction d'eau}

M. ANDRÉ souligne que deux grands types de mesure de débits se dégagent : les mesures par ultrasons et les mesures électromagnétiques.

Il a constaté une grande divergence dans les pourcentages d'erreur annoncés par les utilisateurs (10 à 20\%) et par les constructeurs $(0,2 \%$ environ).

Il souligne l'importance des enjeux financiers d'une mesure précise des débits facturés et d'une gestion optimale des réseaux (problème des fuites).

Il fait deux suggestions pour les études futures :

— pour les débitmètres à ultrasons : il faudrait faire des mesures moins intrusives ;

— pour les débitmètres électromagnétiques : on a toujours utilisé la règle qui veut que le débitmètre doit être précédé d'une conduite droite de longueur égale à 5 diamètres. II 
faudrait vérifier cette règle plus précisément (en particulier voir sa dépendance en fonction du débit et du type de singularités).

M. WESFRIED suggère l'utilisation des débitmètres à vortex dans les conduites d'adduction d'eau. M. ANDRÉ y oppose les problèmes de perte de charge et d'interférence entre les fréquences propres de la structure et celle des vortex.

M. LECOFFRE suggère une méthode optique de mesure de débits.

M. ? ? pense que les critiques contre les débitmètres à ultrasons sont injustifiées car les erreurs de mesure sont dues à un mauvais montage de l'appareil. M. ANDRÉ rappelle qu'il n'est pas toujours facile de positionner précisément le débitmètre sur les conduites industrielles. Mais il reconnait qu'il faudrait aussi améliorer les contacts entre les constructeurs d'appareils et les entreprises chargées de les installer.

\section{Bilan de la $2^{\mathrm{e}}$ journée : Débitmétrie dans les procédés industriels}

M. DELHAYE souligne que les thèmes abordés pendant cette journée étaient très divers et tient à revenir sur 3 points : - le besoin éventuel d'étalonnage en régime transitoire monophasique ;

- les normes: les français sont peu présents dans les groupes de normalisation internationaux et c'est un problème réel ;

- le manque de structure pour aider à l'industrialisation des procédés nouveaux mis au point par les laboratoires.

\section{Défis - Perspectives}

Ces éléments ayant été largement abordés précédemment, les participants ont peu de choses à rajouter.

M. Delhaye énonce deux défis à relever :

- la perfusion parentérale ;

— la débitmétrie pétrolière. 